\title{
Chinese Students in UK Higher Education: Exploratory Research into Chinese Postgraduate Students' Participation in English Dialogic Classroom Activities
}

\author{
Shirui Chai \\ University of Bath
}

\begin{abstract}
Due in no small measure to its rapid economic advance, China has recently found itself enmeshed in a plethora of relationships involving diverse cultures, languages and ethnicities. Consequently, education at one of the higher echelon world-renown universities has become the goal of countless Chinese students. Since existing research suggests that as much as one-fifth of the planet's population have English as their first language, it is entirely predictable that numerous Chinese students would opt to complete their education in the United Kingdom. Hence the startling elevation in the numbers of Chinese nationals attending UK universities over the past decade.

The objective of this study is to focus on the academic experiences of Chinese overseas students at a British university, with specific reference to their intra-classroom dialogic participation. In pursuit of the research goals, concepts of community of practice and dialogic teaching and learning are harnessed for the conceptual structure. The investigation outlined herein is qualitative and as such employs data collection methodology which includes preliminary observation, non-participant observation and semi-structured interview techniques. Data is analyzed thematically, as the coding of fieldwork notes. It is intended that the research will contribute to a revisiting and reassessment of dialogic learning and teaching. Furthermore, it is anticipated that the results will assist those international students for whom English is a second language seeking to complete their higher education at a British university. Whilst the emphasis of the current study is on student participation from a dialogic perspective, it is hoped that future work might be conducted into alternative aspects of student participation, such as critical thinking and reading abilities.
\end{abstract}

Key words: Community of Practice, Dialogic Learning, Classroom interaction and participation, and Chinese students studying abroad. 
 \\ STENAEDUCATION \\ 27-29 March, 2020 \\ Oxford, United Kingdom}

\section{Introduction}

Due to the rapid expansion witnessed in China's economy over recent years, for many hopeful students an overseas university education is now a realistic option. Since English is the lingua franca of international conferences, the media and academic journals, attendance at a British university is an obvious choice. One-year masters programmes in the United Kingdom are popular, during which students' work is evaluated in a variety of ways. One such means is through the establishment of scenarios wherein students are required to work cooperatively in the production of a piece of work. To be successful in such tasks, student participation is a necessary skill which students are required to acquire and utilise in these group-based activities. Learning environments such as lectures, seminars and workshops all present opportunities for students to interact, debate and engage with fellow students. This study aims to explore, from a dialogic perspective, the participation of Chinese students in these learning opportunities at one university in the United Kingdom.

This paper will commence with an outline of the research questions and objectives. Section 3 describes the research context, and Section 4 addresses the issue of research strategy and research design. Detailed discussions of two respective theoretical frameworks are contained in both Section 5 and Section 6, whereas Section 7 pertains to methods of data collection. Section 9 relates to ethical issues and the final part, Section 10, contains the conclusions. Contributions and future works are referenced at the end of the paper.

\section{Research Purposes and Questions}

The objectives of this research are two-fold:

a) An investigation into the impact of dialogic learning experiences on Chinese postgraduate students' motivations to participate in academic practices.

b) An exploration of the development and evaluation of Chinese postgraduate students' abilities to participate in their discipline community of practice.

There exists one overarching research question in this context. To wit, whether dialogic learning experiences in higher education institutions in the United Kingdom facilitate the full participation of Chinese students in the academic practices and activities of their chosen discipline. Thus, the study intends to explore:

a) Whether prior experience of dialogic learning motivates Chinese students to further participate in academic dialogic learning; 
 \\ STENAEDUCATION \\ 27-29 March, 2020 \\ Oxford, United Kingdom}

b) The extent to which opportunities for participation in academic activities are realised for Chinese postgraduate students at universities in the United Kingdom.

\section{Research Contexts}

The location for the research is a British university and the study centres on a group of Chinese students enrolled in a specific postgraduate one-year programme for the academic year 2018/19. English is the medium of instruction at the university. According to the university's statistic records Chinese international students constitute $90 \%$ of the course registration for this programme. Thus, whilst the sample size is small, the subject represents the majority grouping on the programme. The course outline, as described on the university website, indicates that in addition to a dissertation, students are required to successfully complete ten course modules. Assessment for many of these components consists of $30 \%$ coursework, which might relate to either individual or group work, and $70 \%$ examination-based. There is a weekly lecture and a fortnightly workshop or seminar. The former is very much led by academic staff members. However, there exist many opportunities for students to discuss issues relating to the course outside the lecture room. This can take the form of either group discussion amongst students themselves or one-to-one student-lecturer meetings.

\section{Research Strategy and Design}

The methodology employed in this study will be qualitative and interpretive. This will be useful in exploring the anticipated multidimensional nature of the realities and perceptions which this research will investigate. I base the approach on the principle that knowledge is acquired through interfacing and engagement. The interpretive stance is thus preferred since, according to Ritchie et al. (2013), it provides a means of exploring the world through an interpretation of social interactions. For the purposes of the current study, the interpretive approach will facilitate comprehension of participant's perceptions and experiences which will serve to further the study's key objective. To wit, the disclosure of Chinese students' authentic learning experiences at universities in the United Kingdom. Furthermore, the interpretive approach allows for plural outlooks and renderings of reality. Willis and Jost (2007) maintain that the interpretive approach allows those who seek to investigate a group of students to understand how a particular phenomenon is socially constructed (Willis and Jost, 2007). I aim to investigate the realities inter-subjectively, both from students' own experiences and in a particular culture context.

Based on the research goals outlined herein, a qualitative methodology is most appropriate for this study. As highlighted by Maxwell (2012), this methodology relies 
 \\ SLNAEDULATION \\ 27-29 March, 2020 \\ Oxford, United Kingdom}

upon linguistic rather than numerical data and thus the current study is able to focus on subjects' experiences and interpretations thereof. Rather than aiming for a simple hypothesis confirmation, the research anticipates uncovering new insights from the data. In other words, I seek an enriched appreciation of students' experiences and, moreover, to address the research questions from the standpoint of participants themselves. The overall approach is exploratory not confirmatory, in addition to being both qualitative and interpretive. Consequently, it is anticipated that original and thought-provoking findings will be delivered.

The exploratory character of this research relates to a thus far unarticulated or unclearly defined problem. According to Maxwell (2012), exploratory research is particularly appropriate for areas which have been the object of only limited research. Furthermore, as Rowley (2012) clarifies, exploratory research can pave the way for future study through its inherent capacity for gaining fresh insights. Exploratory research design is therefore suitable in the current context due to the dearth of research in the field under consideration. It is thereby intended that the research will contribute significantly to the currently underexplored study of Chinese postgraduate students' academic experience and perspectives in UK higher education, with particular reference to dialogic learning and classroom participation. It is expected that this investigation will engender unique concepts and hypotheses which may well form the basis of future inquiry.

Despite all the benefits of exploratory research outlined above, this mode of research design nevertheless possesses inescapable limitations and disadvantages. As Maxwell (2012) points out, typically the sample sizes employed in this type of research are restricted. Consequently, research results are specific to this study concerned and cannot be extrapolated to the wider population. In addition, since exploratory research is inherently a form of research reconnaissance, the answers it produces will only be provisional not definitive. Nevertheless, it is undeniable that its strength lies in its closely allied ability to provide the impetus for future investigations.

\section{Community of Practice}

\subsection{Definitions and Applications}

The theory of Community of Practice (CoP) is usually attributed to Lave \& Wenger's book on the topic of situated learning (Lave and Wenger, 1991). CoP refers to groups of people who share a common interests or activities, the practice of which is enhanced through regular interaction and communication (Wenger, 2011), and learning is viewed as situated-rooted activity (Wenger, 2000). In classroom activities, students can acquire knowledge via interactions, sharing viewpoints and solving problems with group 
 \\ 《EMMEUUCTION}

members. Instead of learning through direct teaching, students gain knowledge through participation and interaction in the context of a target activity.

It is worth considering the different types of knowledge which students can acquire (Brown and Duguid, 1991). Knowledge can either be "sticky" and "leaky" or both. "Sticky" knowledge is that which relies upon a certain group of people, such as the highly technically skilled people. This type of knowledge is highly valued, but it is not easily transferred or gained. "Sticky" knowledge is difficult to disseminate (Hoadley, 2012). In contrast, "leaky" knowledge is easy to understand and disperse, irrespective of proficiency (Hoadley, 2012). This research regards academic knowledge as "sticky" knowledge that can only be understood by those who major in a particular discipline. In contrast, the skills that needed to participate in classroom activities are regarded as "leaky" knowledge. When students are allocated classroom activities, each one is required to gain the necessary skills to communicate, interact and participate. The "leaky" knowledge required for participatory skills can be generally applied to every subject.

It is conceivable that students who allegedly possess a certain knowledge then fail to apply that body of learning outside the context in which they acquired it. This is exemplified by students who study Law at a university within the United Kingdom but are unable to practice law in China due to differences between the legal systems in the respective countries. Another example is the use of English. In Dearden's research, it indicates that, according to a recent report from the British Council and Oxford University, "there is a fast-moving worldwide shift from English being taught as a foreign language to English being the medium of instruction for academic subjects" (Dearden, 2014). However, a significant body of research indicates that simply teaching in English neither permits students to acquire academic knowledge nor automatically to improve their level of English. It is suggested that schools and universities should incorporate intercultural opportunities and linguistic enhancement activities within their courses (Barnard, 2014). Classroom practice that includes opportunities to practise speaking and discussing in English enables students to understand academic subjects taught in English (Tan and Lan, 2011), thereby making study at UK universities an appropriate choice for international students wishing to learn naturally and authentically.

With regards to process-based definitions, Lave and Wenger (1998) describe CoP as a constant learning process where knowledge is generated, applied and reproduced (Lave and Wenger, 1998). The theory of legitimate peripheral participation (LPP) is evident during this process. Existing literature regarding LPP has informed the creation of a usable model. Figure 1 indicates that how learners shift during the learning process. 

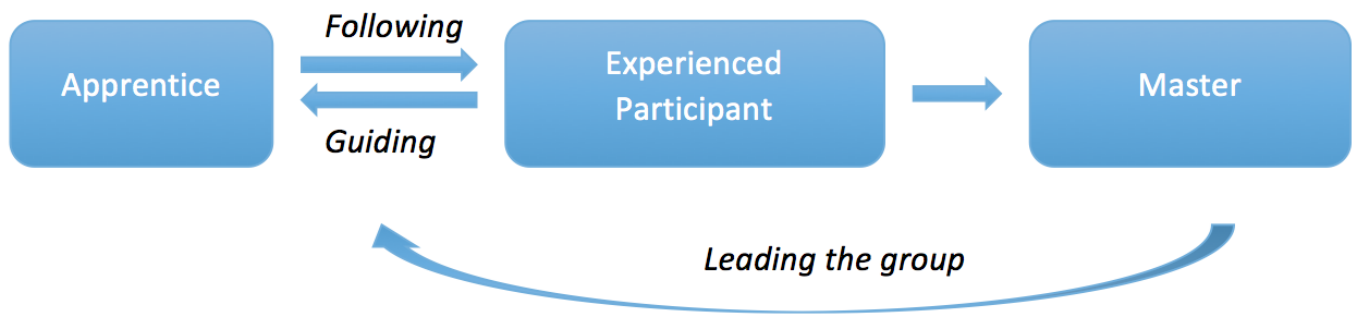

Figure 1. Process of Legitimate Peripheral Participation (LPP)

Apprentices must acquire expertise as they progress towards complete participation. During this journey they frequently benefit from assistance or guidance. On the other hand, experienced participants are more familiar with the skills they are in the process of learning, whereas masters are the acknowledged leaders in the group and constitute the experts in the field. As regards CoP, learners need access to become experts. In this they might consider themselves to be members in a community or aspire themselves to become experts enhanced practices (Lave and Wenger, 1999). This research regards dialogic participation as a means to acquire the knowledge and skills that students need to practice in LPP. In a group activity, students can function as apprentices, experienced participants and masters according to their roles. The extent to which they manage to employ their silks/knowledge in order to participate in dialogic activity is the information which this study intends to locate.

CoP can be identified in contexts where elements existing in the group potentially support CoP. Wenger and Lave (1998) identify three key learning elements in CoP and the literature regarding $\mathrm{CoP}$ has informed the creation of a model. Figure 2 describes the elements that apply to support CoP.

\begin{tabular}{|l|l|l|}
\hline Elements & Connections & Technical supports \\
\hline Mutual Engagement & $\begin{array}{l}\text { Interactions between group } \\
\text { members }\end{array}$ & $\begin{array}{l}\text { Opportunities provided for people to } \\
\text { experience exchanges during an } \\
\text { event }\end{array}$ \\
\hline Joint Enterprise & Common goals and missions & $\begin{array}{l}\text { Supporting people to join an activity } \\
\text { in common contents }\end{array}$ \\
\hline Shared Repertoire & $\begin{array}{l}\text { Ways of communicating work in } \\
\text { the context of group }\end{array}$ & $\begin{array}{l}\text { Skilled information that is used by } \\
\text { the community in its practice }\end{array}$ \\
\hline
\end{tabular}

Figure 2: Connections and technical supports of COP 
 \\ CNAEUULATION \\ 27-29 March, 2020 \\ Oxford, United Kingdom}

Mutual engagement refers to interactions between group members. It is the collaborative relationship that group members create through participation in community. Body language is regarded as an evidence of participation, wherein students might use gestures to convey their viewpoints. Thus, the term "interaction" in this research refers to face-to-face connections. Joint enterprise is the common goal and mission in practices. Group members constantly negotiate and re-negotiate, and possess similar key characteristics in their educational backgrounds and current personal situations (Lave and Wenger, 1998). Moreover, possession of a common repertoire is vital. Not only must students possess appropriate shared tools to practice, but they also need to demonstrate mutual, apposite modes of participation.

Chinese students studying at UK universities must attend regular, compulsory modules. This academic milieu provides opportunities for students to be mutually engaged, forming common bonds founded upon a shared experiences and understandings which serve to unite them as a group. Learning environments provide opportunities for group members to generate a sense of ownership of their learning along with feelings of belonging and commitment to the group. This constitutes the ideal context for the generation of knowledge.

\subsection{Critics and Challenges}

Whilst CoP as a concept has been widely used in many studies, recent research has emphasised its potential challenges and limitations. Firstly, Amin and Roberts (2008) argue that $\mathrm{CoP}$ is not suitable for all forms of situated learning, such as creativity based communities and virtual learning communities (Amin and Roberts, 2008). For example, the Nobel Prize winners would not share their practice with others until after receiving the award. In addition, online learning does not allow learners to have physical responses and interactions. Furthermore, the $\mathrm{CoP}$ notion assumes that learning takes place in a single social setting, whilst in reality learning is more likely to occur across contexts and within multiple social spaces (Fuller, 2013). Fuller and Unwin found that the learners who participated in multiple social settings enjoyed fuller learning experiences than those learners who participated in single settings (Fuller et al., 2005). Learning is constituted and reconstituted by real people in the real world, something which the CoP concept tends to overlook.

Secondly, Hodkinson \& Hodkinson (2004) emphasise that authentic participation should recognize all valuable contributions made by all participants. Thus, engaged learning should highlight the role of co-participation rather than a model focused on individual contributions (Hodkinson and Hodkinson, 2004). The apprentices can bring their own expertise and experiences to classroom activities, and thus the theory of LPP should acknowledge apprentices' perspectives and recognise and include their potential contributions. Dunston (2009, p.40) et al., also argue that all participants in CoP should 
 \\ STEMEDUCATION \\ 27-29 March, 2020 \\ Oxford, United Kingdom}

be seen as "necessary, expert and negative co-participants and co-partners" (Dunston et al., 2009). Furthermore, Kerno (2008, p.69) points that CoP has limitations due to "time constraints, organization hierarchies, and regional culture" (Kerno Jr, 2008). Kerno believes that although learning takes time, many organizations cannot accommodate a prolonged and sustained development of a community of practice. Learners cannot maximise their learning outcomes over a short period of time. In addition, a pre-existing organisational hierarchy is often followed by members of a community of practice (Kerno Jr, 2008). Group members might possess too great a concern for maintaining the hierarchy orders during the learning, which can negatively affect a CoP.

Finally, Danie et al., (2013) indicates that a challenge in CoP is a key function of teaching. In Wenger's theory of CoP, teaching is described as an emerging role in the community of practice. However, in addition to 'emerging' group members, teaching should help to 'develop' members' learning abilities and to 'engage' them in practices.

Despite these criticisms, I acknowledge that CoP theory is entirely appropriate in guiding this research. Firstly, $\mathrm{CoP}$ is useful when it allows members adequate time to engage in activities (Kerno Jr, 2008). The one-year postgraduate programme which forms the context for this study provides sufficient time for students to learn collaboratively. Whilst students are assessed individually at the close of the academic year, there also exists a group-based assessment which is measured in terms of the outcome of group work. Success is based upon effective interactions and collaboration. This research aims to explore whether the constitution of classroom activities allows all members to participate on an equal basis. In conclusion, the concept of $\mathrm{CoP}$ is one of the theories guiding this research.

\section{Dialogic Teaching and Learning}

Dialogic teaching and learning refers to a practical teaching theory which positions dialogue in a centralized location. In dialogic teaching, teachers and students are expected to have equal opportunities to express their views. The theory of dialogic teaching and learning focuses on investigating the moment of dialogue between learners, in addition to how learners are located and constituted by different contexts. The theory explores the individual learning process from learners' the perspective of discourse and reflection. Dialogic learning aims to improve the quality of learning by increasing students' interactions, participation and engagement. 


\subsection{IRE V.S. IRF}

Different modes of classroom interaction have different impacts on students' learning (Skidmore and Murakami, 2016). Compared with dialogic teaching, traditional classroom teaching denotes monologic interaction. Traditional teaching is the pedagogical style in which teachers monopolised talking in the classroom. In teacherled contexts, student are expected to provide correct answers to questions, but otherwise have limited opportunities to develop their understanding of the knowledge (Applebee et al., 2003). Thus monologic interaction is considered to restrict students' critical thinking (Waring, 2009). In contrast, the concept of dialogic learning allows students to play an active and engaged role in a situated learning environment. Through a process of dialogic interchange with group members, dialogic learning develops a personally constructed understanding of learning choices (Lyle, 2008). Research in the field of dialogic teaching and learning has indicated the importance of interaction between learners and their peers as facilitative of learning acquisition by making knowledge comprehensible through negotiation (Varonis and Gass, 1985). The key point of dialogic learning is to improve students' learning ability and outcomes by encouraging total group to participation and to dialogical engagement.

The literature regarding dialogic learning has informed the creation of a model. Figure 3 describes the comparisons between traditional teaching and dialogic teaching. Wells (1993) makes dramatic contributions in term of a re-evaluation of the IRF (initiationresponse-feedback) sequence, a traditional structure in classroom teaching. Instead of giving a direct and immediate evaluation to students, Wells (ibid) promotes the notion that open questions and follow-up responses from teachers allow students to contribute more thoughtfully to classroom discussion. Specifically, these supplementary questions can supply illumination, elucidation and exemplification (Wells,1993). A comparison between traditional IRF and progressive IRF is presented in Figure 3.

\begin{tabular}{|l|l|l|l|}
\hline & $\begin{array}{l}\text { Traditional teaching } \\
\text { and response }\end{array}$ & $\begin{array}{l}\text { Dialogic teaching and } \\
\text { response }\end{array}$ \\
\hline I-initiation & Teacher posts a question & I-initiation & Teacher posts a question \\
\hline R-response & $\begin{array}{l}\text { Student answers a } \\
\text { question }\end{array}$ & R-response & $\begin{array}{l}\text { Student answers a } \\
\text { question }\end{array}$ \\
\hline F-feedback & $\begin{array}{l}\text { Teacher gives direct } \\
\text { response, such as } \\
\text { "correct" }\end{array}$ & F-follow up & $\begin{array}{l}\text { Teacher gives a follow- } \\
\text { up response or an } \\
\text { additional open question }\end{array}$ \\
\hline
\end{tabular}

Figure 3: Traditional Teaching V.S. Dialogic Teaching 
 \\ LCNAEDULATION \\ 27-29 March, 2020 \\ Oxford, United Kingdom}

In relation to different modes of classroom interaction, Bakhtin points that there exist two different classroom discourses, namely, authoritative discourse and internally persuasive discourse (cited by Skidmore, 2000). Authoritative discourse is teacher-led dialogue and test-like questioning. It treats students as knowledge receivers and therefore it limits their active participation (Moore, 2012). Conversely, research suggests that internally persuasive discourse regards students as the key constituent in classroom discourse. This approach is central to the concept of dialogical instruction (Nystrand, 1997). Student-led classroom discourse is characterized by open questions. Students are encouraged to explore follow-up questions. Dialogic discourse removes the emphasis away from the passive receipt of knowledge and towards the improvement of students' scholarly proficiencies. Hence, persuasive discourse focuses on classroom interactions and students' participation.

\subsection{Classroom Interaction and Participation}

The theory of dialogic teaching and learning recognises that the quality of classroom interaction influences learning (Ingram and Elliott, 2014). Turn-taking is a means of organizing conversations during classroom activities, and it is an important way of encouraging learners' interactions and participation (Sacks et al., 1974). Turn-taking is a crucial method which can be employed to actively involve students in class-based pursuits. This research focuses on the order in which different members in a group speak and provides a moment-by-moment construction of this spoken turn-taking. Sacks et al., (1974) indicates that taking a turn at talk not only can present learners' understanding of the knowledge, but also provide evidence of conversation data (Sacks et al., 1974). They maintain that the allocation of space turning is interconnected within the construction of group talk. Group discussions are forums well-suited to frequent transfers of speakership (Sacks et al., 1974). Thus, the group discussion is a key ingredient in classroom participation. The classroom interaction can either take the form of dialogic communication or non-verbal commination. Dialogic communications can be adopted by different members and in different orders; non-verbal communication can manifest itself as roles in organizing group activities, such as making notes, arranging meetings or gesticulating during interactions.

Students' participation and classroom interaction can be grouped into three categories: behavioural engagement, emotional engagement and cognitive engagement (Fredricks et al., 2004). In classroom activities, behavioural engagement denotes students' involvement in learning activities, including attention levels, attempts at asking questions, contributions to arranging group meetings and active participation (Skinner and Belmont, 1993). Emotional engagement is related to students' affective reactions during activities, such as interest levels. One example is where a disagreement occurs and the 'argument' between group members affects the outcome (Connell and Wellborn, 1991). Finally, cognitive engagement encompasses students' psychological 
 \\ SILNAEDUCATION}

27-29 March, 2020

Oxford, United Kingdom

investment during the learning process, such as the skills the learner intends to develop and the knowledge they aim to acquire (Lamborn et al., 1992). Those three engagements are interdependent. Additionally, Nystrand and Gamoran indicate two different classroom engagement types. Respectively, these are procedural engagement and substantive engagement. The former relates to classroom rules and regulations, whilst substantive engagement refers to a sustained personal development in terms of an appreciation for learning (Nystrand and Gamoran, 1991). Procedural engagement has direct links to students' achievements, including academic scores and degree levels. Substantive engagement takes place through a negotiation process between group members. In the process of substantive engagement, students can demonstrate a clearer understanding of the activity via collaborative learning and participating with others. Those are believed to have a more positive impact upon learning. Dialogic learning is treated as one of the substantive learning experiences that have enhanced effects on students' academic achievement.

This research aims to investigate dialogic learning experiences which can help students' classroom participation. It seeks information as to the kinds of skills students gain when they interact with others, whether students alternate between roles, and what determines their participation in group activities. Moreover, this research aims to assess how students manage learning problems such as disagreements during group activities. Finally, this research anticipates evidence that dialogic learning experiences result in students' full participation in academic activities. In summary, the goal is to explore the extent of students' practical engagement in class-based activities and the effects of dialogic learning on learners' interactions and participation levels.

\section{Data Collection and Time Plan}

There are three distinct phases in the data collection associated with this study. Specifically, these are preliminary observations, non-participant observations and semi-structured interviews. Students will be monitored throughout the 2018/19 academic year, although the preliminary observations will be restricted to the first trimester, occurring over a five-week period in the Autumn and Winter of 2018-2019. Non-participant observation will be scheduled over an eight-week period during the second semester, during Winter/Spring 2019, and visual recording will be applied. The final phase constitutes semi-structured interviews which require each interviewee to participate in two interviews. The initial interview will take place during the early stage of the non-participant observation phase and the second interview will occur towards the completion of this phase. Audio recording will be utilized during this phase. 
 \\ CNAEUULATION \\ 27-29 March, 2020 \\ Oxford, United Kingdom}

\section{Step 1: Preliminary Observations}

During this stage it is anticipated that the master's programme lectures will be observed over a five-week period during the first semester, which is due to begin in November 2019. Through preliminary observation of the lectures, prospective participants can be selected. The criteria of selection are four to six Chinese postgraduate students whom English is a second language. The preliminary observation will also provide an opportunity to observe the extent to which students are occasioned the opportunity to participate in dialog activities. The module selected for this stage consists of $70 \%$ examination assessment and 30\% coursework. The university website states that students are allocated seminar groups for this module. Regular fortnightly seminars facilitate discussion amongst students of coursework content and any difficulties arising therein. The coursework itself is produced and consequently assessed on an individual basis, although students are at liberty to consult and cooperate with each other should they wish to do so. Thus, the course outline on the university's website suggests the existence of multiple opportunities to engage in information exchange and academic interaction, at least in theory. Those selected as participants for this study will be involved in both direct observations and interviews.

\section{Step 2: Non-participant Observations}

During the non-participant observation, I will attend learning activities for the purposes of objectively observing participants and will not participate in the learning activities or engage with the participants at this stage. This will facilitate the gathering of nonverbal information such as participants' exhibited emotions. Furthermore, it permits the observation of unanticipated interactions and group dynamics, including modes of communication, time allocated by students to each target task and techniques employed by students during class-based activities.

In non-participant observations, the intention is to observe and record the skills or techniques employed by group members in classroom activities. Visual recordings are used and field notes are taken during each observation session. The visual recording will record a group activity with a focus on the selected participants' behaviour and engagement. The former permit subsequent moment-by-moment analysis of students' behavioral changes during classroom interactions. In addition, field notes are taken in situ at key moments which will provide depth and detail in later data analyses.

The target participants are a group of between four and six Chinese international students in a master programme. Additionally, it is necessary to observe individuals who interact with the target participants during the observations. This might either be other students or the academic staff. The focus during these observations will be the engagements between the target participants, and their dialogic interactions. It is intended that group dialogic interactions will be observed, with a focus on the responses 
 \\ SIENAEDUCATION \\ 27-29 March, 2020 \\ Oxford, United Kingdom}

of the target participants. Others' responses are relevant, but subsidiary. The evidence from non-participant observation takes the form of first hand notes relating to preprepared research questions.

\section{Step 3: Semi-Structured interviews}

The final stage consists of semi-structured interviews which require participants to respond to a series of pre-prepared, in-depth questions in addition to any follow-up questions. Since this is qualitative research, most questions will be of the open-ended variety. I anticipate to use of guidance during the interviews, in which key points are isolated, questions are devised and timings are established. Data obtained from the earlier observations will inform the content of the pre-prepared interview questions, whilst participants' responses to these enquiries will determine any follow-up questions. These latter queries will take on a more exploratory character, concentrating on participants' personal experiences of dialogic learning and activities.

The sample group will consist of between four and six postgraduate students selected during the previous observation stage. It is intended that the first of the two interviews in which each interviewee participates will be held at the start of the semester, and the second towards the final part. The inclusion of two interviews is crucial since this permits the chronicling of any possible discrepancies or changes in students' attitudes between during the learning period. Audio recording will be employed at this stage since it will permit me to focus on the interview process itself, although limited field notes will also be taken during the interviews. These notes can help ensure the logicality and fluency of the interviews. Data from the interviews will form the basis upon which later research questions are addressed.

\section{Ethics}

There are several ethical considerations that require addressing at this stage. The first of these relates to the ethnic and linguistic backgrounds of participants. These characteristics were a determining factor in the selection of candidates for inclusion. However, all necessary measures will be taken to ensure no subject is detrimentally impacted by agreeing to participate in the research. The second ethical safeguard relates to the provision of all participants with an information sheet prior to the commencement of data collection, and their notification that they have the right to withdraw their participation at any stage. I am prepared to discuss the aims, methods and process etc. of the research with participants if requested, to ensure that they fully understand the nature of the study. No visual or audio recordings will occur without specific permission from those who might be involved. Finally, participant identities will be anonymised through the employment of pseudonyms. 
 \\ STENAEDUCATION \\ 27-29 March, 2020 \\ Oxford, United Kingdom}

\section{Contributions and Future Directions for Research}

Whilst current thinking indicates that classroom dialogue facilitates elevated levels of student participation in learning, it is significant that this is based on findings regarding studies conducted in the school environment. The effects of dialogic teaching and learning either in a university setting or in a situation where learners are using English as a second language remains comparatively under-researched. In this context, this study intends to assess the effect on the participation of Chinese postgraduate students in academic practices of dialogic learning experiences. Furthermore, the study aims to investigate the progress of these students' participation in their discipline's community.

There are two potential outcomes that this study might achieve. The first involves the prompting of a re-evaluation of dialogic learning. Whereas in theory dialogic learning has a value in that it engages students and promotes their interaction in the classroom, when conditions are altered the effects may vary. Specifically, this study aims to rethink the contribution of dialogic learning within alternative environments. The second possible outcome from the study relates to the increasing numbers of Chinese students attending higher education institutions in the United Kingdom. It is anticipated that this research might identify learning techniques for these students which will enable them to overcome the barriers they encounter when studying abroad.

Future research might take one of two possible paths. Firstly, whilst the current proposed study relates to the effects of dialogic learning on students' engagement and participation, forthcoming research might analyse dialogic learning from alternative stances, including students' reading abilities or critical thinking skills. Secondly, future studies might investigate other influences on student participation. Currently the number of Chinese students attending universities in the United Kingdom and elsewhere is increasing. This study will contribute significantly to an appreciation of their dialogic learning and participation aspects of these experiences. 


\section{Bibliography}

AMIN, A. \& ROBERTS, J. 2008. Knowing in action: Beyond communities of practice. Research policy, 37, 353-369.

APPLEBEE, A. N., LANGER, J. A., NYSTRAND, M. \& GAMORAN, A. 2003. Discussionbased approaches to developing understanding: Classroom instruction and student performance in middle and high school English. American Educational Research Journal, 40, 685-730.

BARNARD, R. 2014. English medium instruction in Asian universities: Some concerns and a suggested approach to dual-medium instruction. Indonesian Journal of Applied Linguistics, 4, 10-22.

BROWN, J. S. \& DUGUID, P. 1991. Organizational learning and communities-ofpractice: Toward a unified view of working, learning, and innovation. Organization science, 2, 40-57.

DEARDEN, J. 2014. English as a medium of instruction-a growing global phenomenon, British Council.

DUNSTON, R., LEE, A., BOUD, D., BRODIE, P. \& CHIARELlA, M. 2009. Co production and health system reform-from re - imagining to re - making. Australian Journal of Public Administration, 68, 39-52.

FULLER, A. 2013. Critiquing theories of learning and communities of practice. Communities of practice. Routledge.

FULLER, A., HODKINSON, H., HODKINSON, P. \& UNWIN, L. 2005. Learning as peripheral participation in communities of practice: a reassessment of key concepts in workplace learning. British Educational Research Journal, 31, 49-68.

HOADLEY, C. 2012. 12 What is a Community of Practice and How Can We Support It? Theoretical foundations of learning environments, 286.

HODKINSON, H. \& HODKINSON, P. 2004. Rethinking the concept of community of practice in relation to schoolteachers' workplace learning. International journal of training and development, 8, 21-31.

INGRAM, J. \& ELLIOTT, V. 2014. Turn taking and 'wait time'in classroom interactions. Journal of Pragmatics, 62, 1-12.

KERNO JR, S. J. 2008. Limitations of communities of practice: a consideration of unresolved issues and difficulties in the approach. Journal of Leadership \& Organizational Studies, 15, 69-78. 
LAVE, J. \& WENGER, E. 1991. Situated learning: Legitimate peripheral participation, Cambridge university press.

LAVE, J. \& WENGER, E. 1998. Communities of practice. Retrieved June, 9, 2008.

LAVE, J. \& WENGER, E. 1999. Learning and pedagogy in communities of practice. Learners and pedagogy, 21-33.

LYLE, S. 2008. Dialogic teaching: Discussing theoretical contexts and reviewing evidence from classroom practice. Language and education, 22, 222-240.

MAXWELL, J. A. 2012. Qualitative research design: An interactive approach, Sage publications.

NYSTRAND, M. 1997. Opening Dialogue: Understanding the Dynamics of Language and Learning in the English Classroom. Language and Literacy Series, ERIC.

RITCHIE, J., LEWIS, J., NICHOLLS, C. M. \& ORMSTON, R. 2013. Qualitative research practice: A guide for social science students and researchers, Sage.

ROWLEY, J. 2002. Using case studies in research. Management research news, 25, 16-27.

SACKS, H., SEBEGLOFF, E. \& JEFFERSON, G. 1974. A simplcst systemalies for tbe oIJuizalion oe turn-taking in conversation. LQ. fIgU4ge, 50, 696-735.

SKIDMORE, D. \& MURAKAMI, K. 2016. Dialogic pedagogy: the importance of dialogue in teaching and learning, Multilingual Matters.

TAN, M. \& LAN, O. S. 2011. Teaching mathematics and science in English in Malaysian classrooms: The impact of teacher beliefs on classroom practices and student learning. Journal of English for Academic Purposes, 10, 5-18.

VARONIS, E. M. \& GASS, S. M. 1985. Miscommunication in native/nonnative conversation. Language in society, 14, 327-343.

WARING, H. Z. 2009. Moving out of IRF (Initiation - Response - Feedback): A single case analysis. Language Learning, 59, 796-824.

WENGER, E. 2000. Communities of practice and social learning systems. Organization, 7, 225-246.

WENGER, E. 2011. Communities of practice: A brief introduction.

WILLIS, J. W. \& JOST, M. 2007. Foundations of qualitative research: Interpretive and critical approaches, Sage. 